\title{
The Chemnitz LogAnalyzer: A tool for analyzing data from hypertext navigation research
}

\author{
ANGELA BRUNSTEIN, ANJA NAUMANN, and JOSEF F. KREMS \\ Chemnitz University of Technology, Chemnitz, Germany
}

\begin{abstract}
Computer-based studies usually produce log files as raw data. These data cannot be analyzed adequately with conventional statistical software. The Chemnitz LogAnalyzer provides tools for quick and comfortable visualization and analyses of hypertext navigation behavior by individual users and for aggregated data. In addition, it supports analogous analyses of questionnaire data and reanalysis with respect to several predefined orders of nodes of the same hypertext. As an illustration of how to use the Chemnitz LogAnalyzer, we give an account of one study on learning with hypertext. Participants either searched for specific details or read a hypertext document to familiarize themselves with its content. The tool helped identify navigation strategies affected by these two processing goals and provided comparisons, for example, of processing times and visited sites. Altogether, the Chemnitz LogAnalyzer fills the gap between log files as raw data of Web-based studies and conventional statistical software.
\end{abstract}

Computer-based studies usually produce log files as raw data. In contrast to paper-and-pencil-based studies, these data are already digitalized. With this approach, behavioral data are recorded automatically and are, therefore, much less expensive than those that must be processed by hand-for example, verbal protocols.

Unfortunately, conventional Web site log file analysis software is designed for the understanding of Web site use for the optimization of Web site design, instead of the analysis of human behavior per se. Correspondingly, many tools for Web log file analysis provide only aggregate statistics for large-scale data sets, rather than measurements of the individual navigation behavior of participants within a psychological experiment.

Fortunately, there exist a few tools for analyzing Web experiments log files. These include STRATDYN (Berendt \& Brenstein, 2001), LOGPAT (Richter, Naumann, \& Noller, 2003), and the Scientific LogAnalyzer (Reips \& Stieger, 2004). STRATDYN and LOGPAT, for example, are based on graph theory and supply quantitative values for the coherence of hypertexts or of navigation strategies. Their analyses require that the participant's navigation behavior can be clearly categorized and that enough data are available. In addition, STRATDYN offers detailed and dynamic options for the visualization of individual navigation paths. The Scientific LogAnalyzer

This article was presented at the Society for Computers in Psychology (SCiP) conference in Minneapolis, November 18, 2004, and was supported by German Research Foundation Grant KR 1057. We thank Tobias Winkler for programming the Chemnitz LogAnalyzer, Jacqueline Waniek for her help in the development of the tool, and Frank Ritter and an anonymous reviewer for helpful comments on earlier versions of the manuscript. Correspondence concerning this article should be addressed to A. Brunstein, Department of Psychology, Program in Cognitive Psychology, Chemnitz University of Technology, D-09107 Chemnitz, Germany (e-mail: angela.brunstein@phil.tu-chemnitz.de)
(Reips \& Stieger, 2004) further allows for the extraction of data collected from on-line questionnaires and forms and has several features of built-in Web-based research methodology. This tool provides processing data for visited nodes, but not for processed items of questionnaires and forms. Although these three tools surpass the conventional tools for log file analysis, they nevertheless provide no or insufficient means of displaying such values as aggregated visiting times or navigation paths, as compared with one or several theoretically founded orders of nodes. Instead, visualizations of navigation paths are arranged according to individual user's behavior and, therefore, vary from trial to trial.

The Chemnitz LogAnalyzer began development in 2001 within the research program "User adapted presentation of information on the Internet." It is written in C++ and is now available as version 2.14 (www.tu-chemnitz. de/phil/psych/projekte/NeueMedien). It helps translate raw Web log files into tables of raw data for use with popular statistical programs in which one participant or node is presented per row. It also provides tools for quick and comfortable visualizing and analyzing navigation behavior within hypertexts. Some of these options, such as the visualization of navigation behavior, are also missing in statistical programs. The tool is user friendly and requires no programming skills. It provides both a quick first survey of data for explorative analysis and statistical values for quantitative analysis. Similar to the tools mentioned above, the Chemnitz LogAnalyzer enables graph-theoretic analyses by providing transition matrices between nodes. In contrast to these tools, it provides illustrations of navigation pattern as matrices (see Figures 1 and 2), instead of graphs or tree structures. Perhaps most important, these matrices are organized according to theoretically founded orders of nodes within a hypertext document, and therefore, they allow the integrating of hypotheses about 


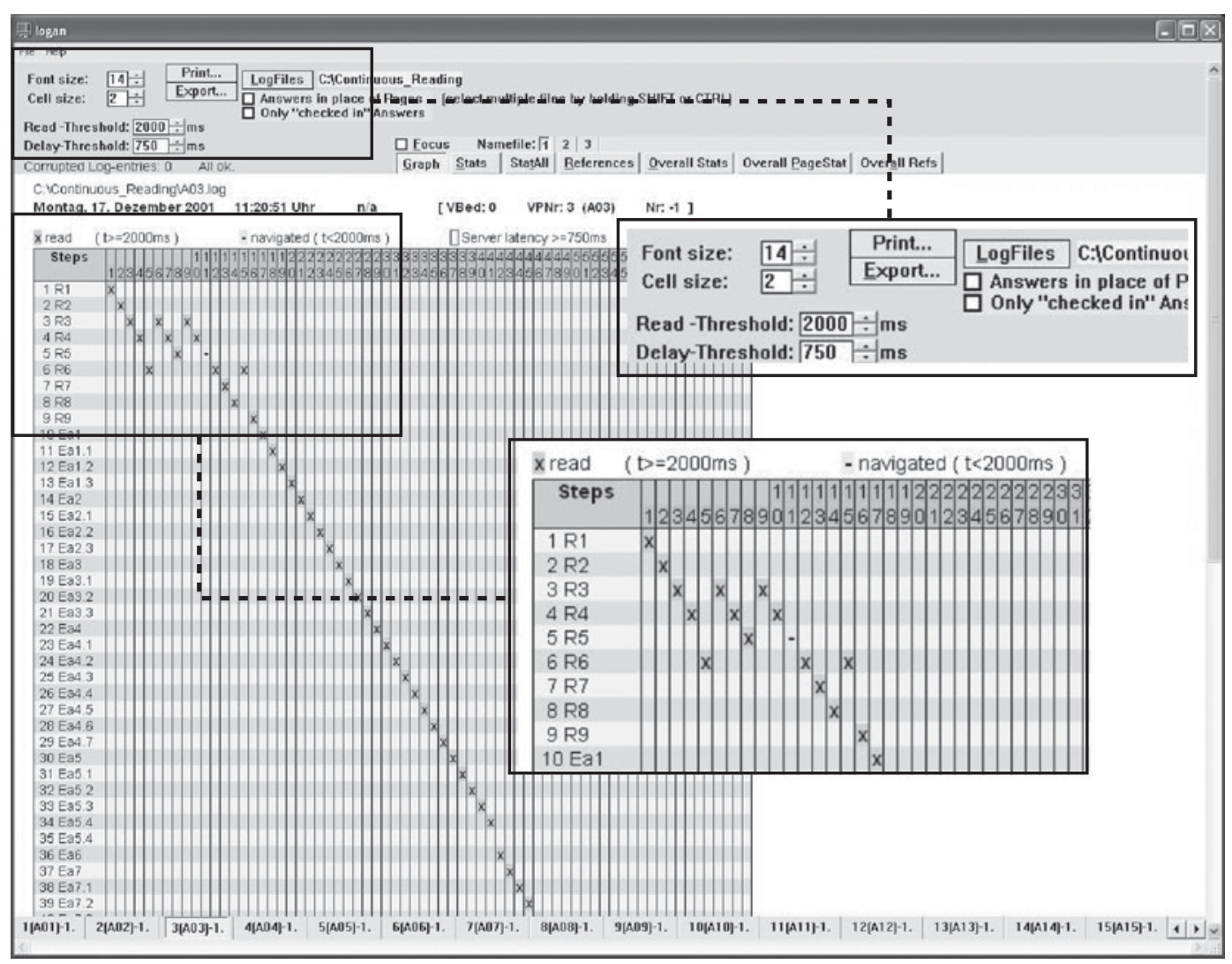

Figure 1. The Chemnitz LogAnalyzer, showing the Graph display for visualizing individual navigation paths.

navigation patterns into the visualization of participants' navigation behavior. Moreover, it can compute the total time for visiting each node and the number of switches between nodes (see Figure 3) for both individual and aggregated data. It also supports an analogous analysis of questionnaire data for hypertext-based learning systems that contain exercises as multiple choice items (see Figure 4). Nevertheless, the Chemnitz LogAnalyzer does not transfer answers for open-ended questions or chosen options from pull-down menus from log files to row data tables, as the Scientific LogAnalyzer does.

To illustrate these navigation patterns, navigation paths of participants, in groups or individually, are shown (see Figures 1 and 2). The figures are illustrations of the large example explained below. A single participant's or a group's preferred linkage structures can also be illustrated: Visiting, reading, and navigation times, number of visited nodes, and switches between nodes are used in the analysis of navigation data per participant or per nodes (see Figure 3). Visited nodes or, alternatively, answers for multiple choice items can be analyzed. Analogously to the nodes visited, the order of treated items can be visualized. Moreover, the tool differentiates between correct and incorrect answers when the log file contains this information. Data are stored in a .txt file and can, thus, be easily exported to most statistic software packages.
There are few requirements for using the tool. The program runs locally on the user's computer, using the .exe format; therefore, there is no need to transmit sensitive data via the Internet. Moreover, the user controls the processing completely. The tool runs only on all 32-bit Microsoft Windows versions. Nevertheless, its results can be viewed independently on other platforms as .txt files. Using the tool requires no complex installation or special resources. To start using the Chemnitz LogAnalyzer, one has simply to download the program and to double click on the corresponding icon. At the moment, it is necessary to have one log file per participant and session in the .log format (see Figure 5). Examples of $\log$ files and format requirements and detailed instructions on how to use this tool can be found in the manual at the program Web page (www.tu-chemnitz.de/phil/psych/projekte/NeueMedien) or in the report of our experimental environment (Naumann, Brunstein, \& Krems, 2005). Log files generated by servers that contain the data for all the participants cannot be analyzed using the Chemnitz LogAnalyzer at the moment without adapting either the log files or the tool.

The program requires node sets containing the names of the nodes within the hypertext in the order of occurrence, in the .txt format. These node sets have to be set up beforehand and contain the sequence of hypertext nodes with which the users' reading sequence is supported to be 


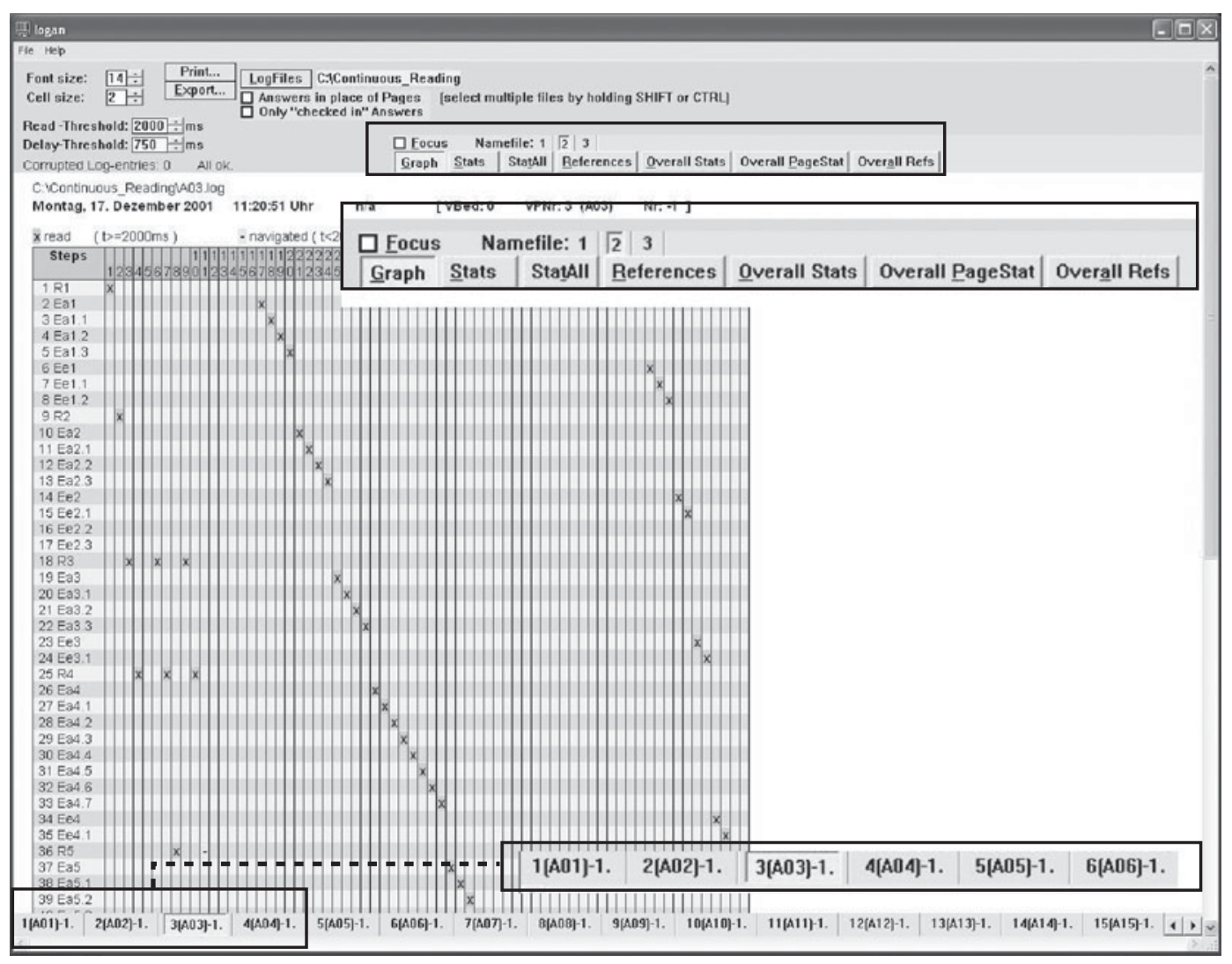

Figure 2. The Graph display for the same participant as in Figure 1, using the format-based node set.

compared with. In contrast to STRATDYN and the Scientific LogAnalyzer, the Chemnitz LogAnalyzer allows the analysis of navigation paths in relation to one or more predefined orders (see Figures 1 and 2: two different orders of nodes for the same hypertext and the same participant). These orders should be derived by processing hypotheses. As a consequence, navigation paths of different users are easy to compare. Node sets are the regulating structure of the nodes being investigated. As such, they are the main instruments for handling all the possibilities when analyzing available log files, and they enable quick and flexible reanalysis of the data for an alternative predefined order of nodes of one and the same hypertext. Corresponding node sets ease the quick identification of navigation strategies.

\section{THE CHEMNITZ LOGANALYZER IN DETAIL}

The Chemnitz LogAnalyzer user interface is divided into two parts (see Figure 1): the тепи at the top of the interface (see the gray part in Figures 1 and 2) and the display at the bottom (see the white part in Figures 1 and 2). Whereas the menu handles necessary settings, the display provides navigation patterns, as well as the results of the different analyses.

\section{Functions of the Menu}

The menu offers, along with settings for display fonts and cell widths, data export and printing and important settings for the $\log$ file analysis. The LogFiles button opens a menu for choosing a single or several log files for analysis.

Furthermore, important parameters for the log file analysis have to be set, such as the specification of the reading threshold, which separates nodes that were read from navigated nodes, as well as the specification of critical server delays. The reading threshold should be chosen depending on the actual hypertext. For instance, reading a specialized text requires more processing time than does viewing a picture or filling in a formula. Server delays may be difficult to handle in Web-based studies, and critical server delays should be chosen on the basis of server and network connection speed. In the navigation paths display, read nodes are marked with a dark blue "x," whereas navigated nodes are marked with a light blue "-." Critical server delays are adjusted by choosing the delay threshold. All nodes presented after that threshold are marked within the visualization of navigation paths with a red box.

One can also choose between analyzing navigation patterns or answers of multiple choice items by clicking the "Answers in Place of Pages" check box. In order to 


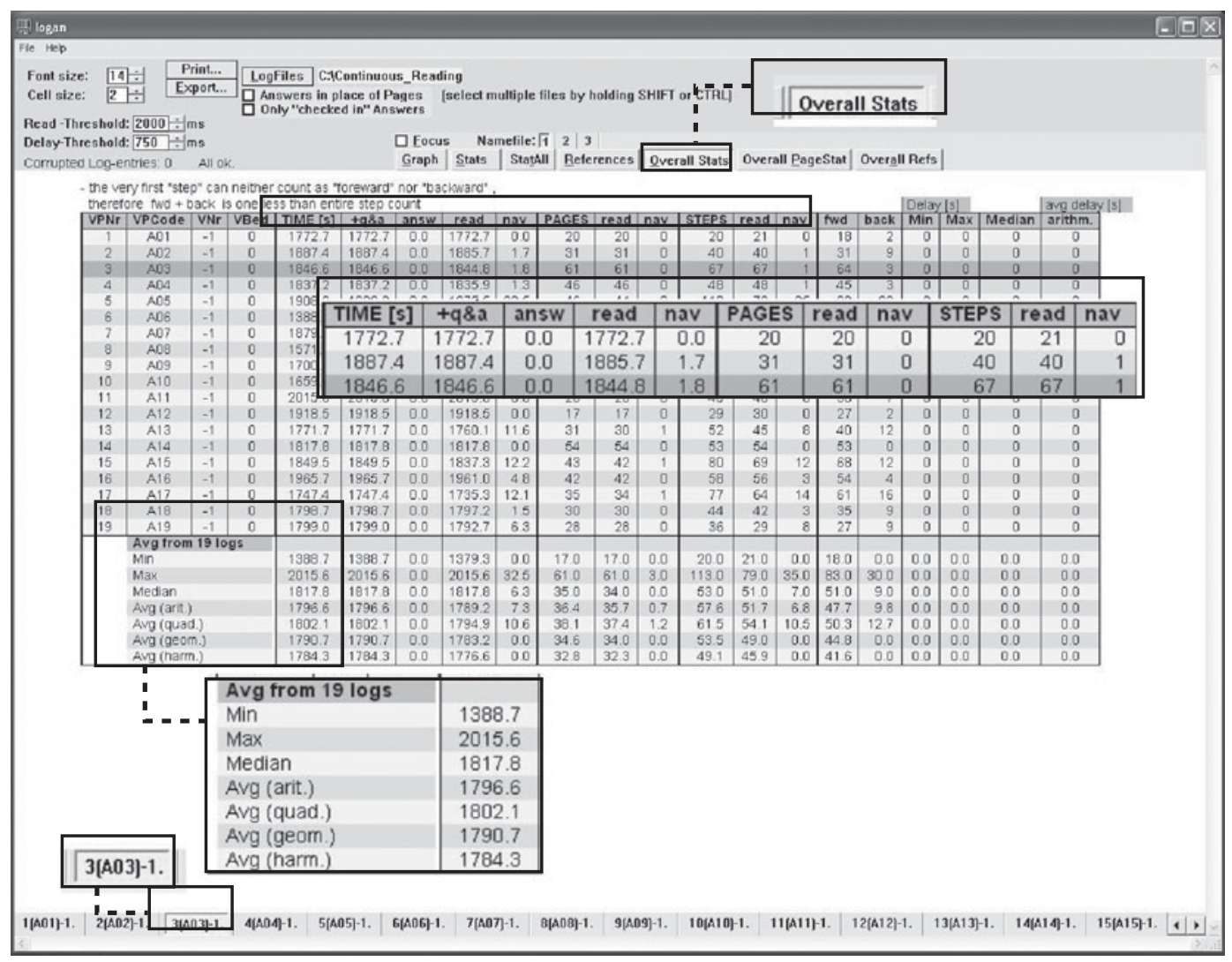

Figure 3. The Overall Stats display.

analyze answers, the same functions are provided as in navigation paths. Within the visualization of answering behavior, correctly answered questions are marked with an "x," and incorrectly answered questions are marked with a "-," as long as the log files provide that information (see Figure 4). Given no information about correctness of answers, all answers are marked with an "x." For the actual study described below, there was a "check" button in the hypertext for controlling given answers. Red boxes in the Graph display indicate that the participants did not control their answers with the check button.

Seven flags on the bottom right-hand side of the menu allow switching among different views of the display below and accessing several sets of information about navigation and answering behavior.

\section{Multiple Displays}

The display provides various visualizations and analyses of navigation patterns. One can choose among Graph, References, and Overall Refs, illustrating navigation patterns, and Stats, StatAll, Overall Stats, and PageStat, presenting statistical analyses. These functions will be explained in more detail next.

The Graph display illustrates the navigation path for 1 participant from the first node visited all the way to the last node visited or, alternatively, the order of multiple choice items answered by this participant. The order of answered questions can be important, with a theoretically based order of exercises within a hypertext-based learning system, for checking whether following that order improves performance. Corresponding to the chosen node set, nodes are arranged as rows of the matrix, and navigation steps are arranged as columns (see Figures 1 and 2). Therefore, the navigation path of 1 participant through the hypertext is displayed from left to right. After choosing the focus function within the menu above, the actual position of the mouse pointer within the matrix is highlighted, and the corresponding statistical values are displayed in the menu. You can switch between paths of different participants via flags at the bottom of the window, which show the name of the log file (e.g., the number of the corresponding participant; see Figure 1, "3[A03]-1" for Participant A03, considered third, and his or her first session).

In addition to individual navigation paths, the References and Overall Refs displays illustrate all switches between nodes, ignoring the time of the individual visits and the order of switches, to highlight prominent navigation patterns for one (References) or for all (Overall Refs) participants. These displays can be used, for example, to create a graphical analysis of behavior.

The Stats and StatAll displays show visiting times, as well as switches between nodes, as a numeric table of visiting times and transition frequencies per participant (Stats) or for all the participants (StatAll). The Stats display also 


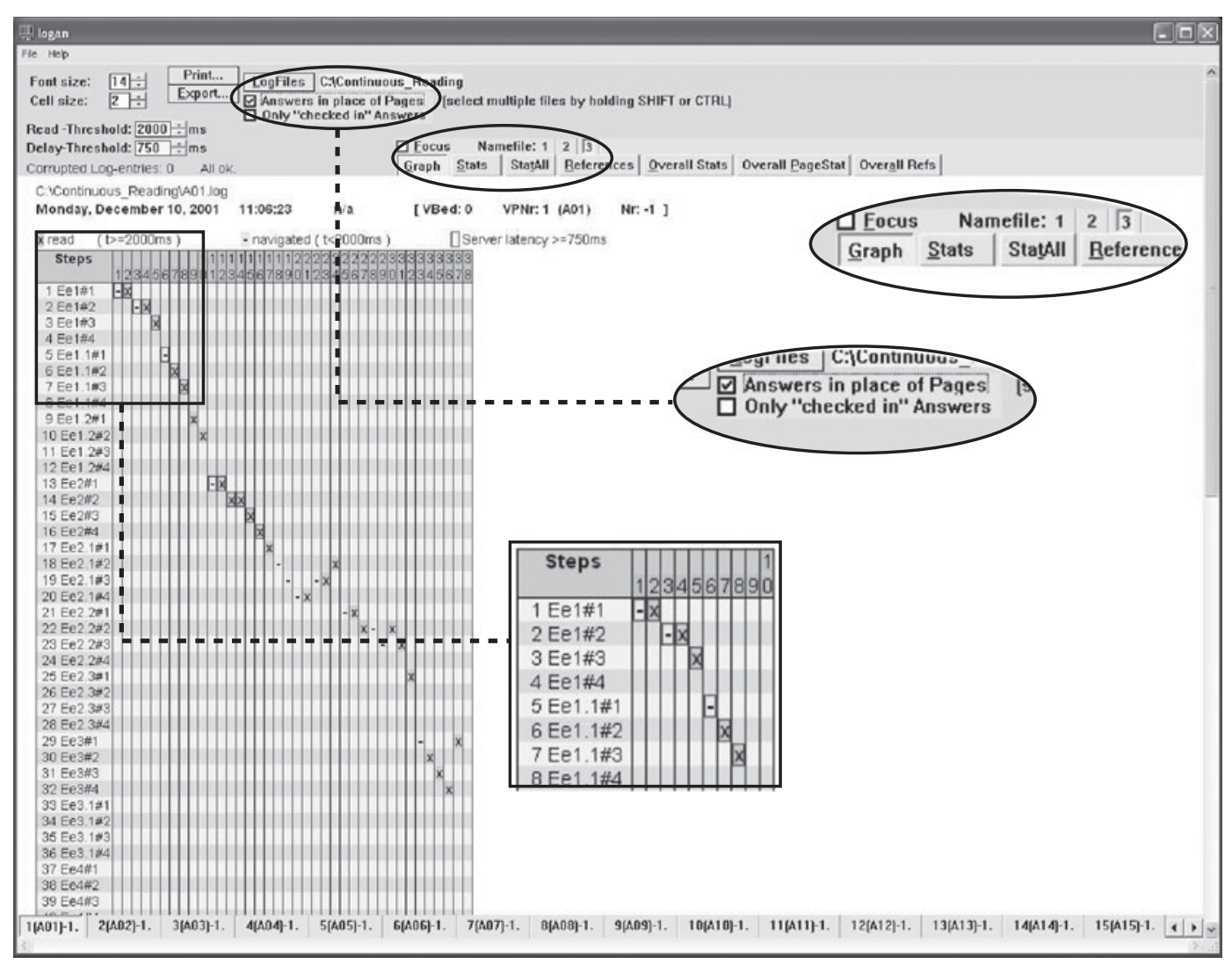

Figure 4. The Graph display for answered multiple choice items.

contains the overall processing time, reading (defined by the reading threshold) and navigation time, and forward and backward switches. As for navigation paths in the Graph display, one can switch between statistical values of different participants via flags at the bottom of the window.
The Overall Stats (see Figure 3) and Overall PageStat displays summarize the results of visiting, reading, and navigation times, as well as switches between nodes, by giving average values per participant (Overall Stats) or per node (Overall PageStat). At the bottom of the Overall

\begin{tabular}{|c|c|c|c|c|}
\hline Continuous & Read & $n g$ & st session & \\
\hline frorn card & card & "start" & & 47182 \\
\hline to card & card & id 44204 & & 47197 \\
\hline Start! & & & & 164786 \\
\hline frora card & card & id 44204 & 164788 & \\
\hline to card & $\operatorname{card}$ & "R1" & & 164806 \\
\hline Button: & card & "R1" & f lag-exarop les & 170406 \\
\hline from card & card & "R1" & & 170408 \\
\hline to card & card & "Ea1" & & 170462 \\
\hline Button: & card & "Ea1" & flag-exercises & 172025 \\
\hline from card & card & "Ea1" & & 172026 \\
\hline to card & card & "Ee1" & & 172063 \\
\hline exercise: & card & "Ee1" & Wr . 1173685 & wrong \\
\hline exercise: & card & "Ee1" & Nr . 117.5098 & correct \\
\hline exercise: & card & "EE $1 "$ & Nr . 2176849 & wrong \\
\hline exercise: & card & "Ee1" & $\mathrm{Nr} .2177463$ & correct \\
\hline exercise: & card & "Ee1" & Nr . 31782.59 & correct \\
\hline Button: & card & "Ee1" & go next & 178381 \\
\hline
\end{tabular}

Figure 5. A piece of a log file out of a Hypercard study analyzed using the Chemnitz LogAnalyzer. 
Stats matrix, means, minima, and maxima, aggregated for all the participants, are displayed.

\section{USING THE CHEMNITZ LOGANALYZER FOR IDENTIFYING PROCESSING STRATEGIES}

As an illustration of how to use the Chemnitz LogAnalyzer, we give an account of one study on learning with hypertext. Brunstein and Krems (2005) investigated the influence of a processing goal on performance and learning outcome. Most Web-based learning systems have had participants either search for specific information within the hypertext or read the text to familiarize themselves with its content. These two modes of behavior drive the analyses below.

The hypertext used in this study was organized in a tightly connected net. Thus, each node could be reached from any other node by, at maximum, two clicks. There were two reasonable and theoretically founded choices of order of nodes for processing the hypertext. One way of processing was to organize travel through the nodes according to the themes of that hypertext, corresponding to the organization of a mental model of the content. The other way was based on the format, in which information was presented as rules, examples, or exercises, corresponding to different learning styles. Both orders were about equally probable for use for navigating through the chapters, because of their netlike organization.

As had been shown by previous hypertext research (e.g., Dee-Lucas, 1996; Dee-Lucas \& Larkin, 1999; Foltz, 1996; Wiley, 2001), we found that participants searching for specific information (searchers) and participants reading the hypertext to familiarize themselves with its content (skimmers) answered detailed questions equally well. Nevertheless, the skimmers, but not the searchers, demonstrated a better application of their knowledge afterward (Brunstein \& Krems, 2003; Brunstein, Waniek, Naumann, \& Krems, 2002).

Using the Chemnitz LogAnalyzer, we investigated whether different processing strategies, corresponding to different processing goals, were responsible for these different learning outcomes. We expected that the participants would prefer two possible kinds of predefined paths to reach their goals. The searchers would use the final questions to order their searching. They would, therefore, navigate through the hypertext according to the presented themes. To find as much evidence as possible, they would stay longer on visited nodes than would the skimmers and would, therefore, visit fewer nodes than would the skimmers within a given time. The skimmers had no external guidance and had to organize their processing of hypertext on their own. Thus, the skimmers would navigate through the hypertext either according to themes or according to the given formats, at chance level. To get a broad review of the information presented, the skimmers would visit as many nodes as possible and should spend less time on singular nodes than would the searchers.

\section{Method}

Forty students at Chemnitz University of Technology (32 females and 8 males; mean age of 23 years, $S D=$ 6 years) participated in this study. They indicated German as their mother tongue, reported having an average of 7 years of English $(S D=1.5$ years $)$, and were familiar with using computers.

We adapted two chapters of the Chemnitz Internet Grammar (www.tu-chemnitz.de/phil/InternetGrammar/) to laboratory conditions, using Hypercard running on PowerPCs. The Internet Grammar is a hypertext-based learning system for English grammar and was developed by Hahn, Reich, and Schmied (2000). The chapters covered the present continuous and present perfect tenses and contained about 75 nodes and about 9,000 words each. Three navigation aids where presented on each page. (1) A content list on the left listed themes or situations with which to use and not use these verb tenses. (2) Flags at the top separated presentation format of rules, examples, and exercises for each situation. (3) Forward and backward buttons at the bottom of each page directed the participants from the first rule node to the last exercise node. In addition to hypertext chapters, the participants processed application tasks, before and after processing the chapters, in a paper-and-pencil version. As application tasks, the participants had to fill in the correct verb form for 21 English sentences.

The searchers were instructed to use the chapters for answering 10 open-ended detailed searching questions and ten multiple choice items presented on paper. The skimmers were instructed to process the chapter in order to familiarize themselves with its content. They answered the same detailed questions after processing the chapters. For instance, one open-ended question asked for the duration of residence for being allowed to say "I am living in Chemnitz." One multiple choice item asked whether "I am working here" means "it is for the length of a fixed project," "it is not clear how long I stay," or "I am new here." The moment of presenting these detailed questions was used to set the independent variables by manipulating the processing goals of the participants.

The participants processed pretests, chapters, and posttests in two sessions lasting $1 \mathrm{~h}$ each. To process the chapters, they were given $30 \mathrm{~min}$ per session. The order of test versions and chapters was balanced out between participants for both processing goals.

As an independent variable, the processing goal of searching for specific information, versus reading for becoming familiarized with the content, was manipulated. Performance on tests and processing times, number and order of visited nodes, and switches between nodes and exercises out of log files (see Figure 5) were collected as dependent measures.

\section{Results}

Performance on detailed questions and learning outcome. The skimmers (in the following, indicated by the index $S k)$ answered more detailed questions $\left(M_{\mathrm{Sk}}=18\right.$ 
out of $\left.20, S D_{\mathrm{Sk}}=2.5\right)$ after processing a grammar chapter than did the searchers (in the following indicated by the index $\left.S e ; M_{\mathrm{Se}}=14, S D_{\mathrm{Se}}=4.3\right)$ on line during processing $\left[F(1,37)=23.83, p<.001, \eta^{2}=.39\right]$. Both groups answered questions comparably well $\left(M_{\mathrm{Sk}}=13\right.$ out of 20 , $S D_{\mathrm{Sk}}=3.2 ; M_{\mathrm{Se}}=12$ out of $\left.20, S D_{\mathrm{Se}}=3.5\right)$, indicating that both groups processed the grammar chapters in detail.

After processing a grammar chapter, the skimmers $\left(M_{\mathrm{Sk}}=+3, S D_{\mathrm{Sk}}=3.3\right)$ but not the searchers $\left(M_{\mathrm{Se}}=-1\right.$, $\left.S D_{\mathrm{Se}}=4.3\right)$, improved their performance on application tasks, as compared with their performance before processing the chapter $\left[F(1,36)=9.21, p<.01, \eta^{2}=.20\right]$.

Navigation strategies. For identifying navigation patterns, we consulted the Graph display in the Chemnitz LogAnalyzer. We created two node sets corresponding to the two hypothesized kinds of predefined orders of nodes of the hypertext - one corresponding to themes or content of an assumed mental model of the content, and one corresponding to different learning styles associated with different formats of information. Therefore, themes (situations in which to use and not use the present continuous and the perfect tenses) were numbered from 1 to 12 and formats were coded as " $r$ " for rules, "ea" for examples (illustrative sentences), and "ee" for exercises (sentences with gaps to fill in the correct verb form). The thematic node set contained codes for $\mathrm{r} 1$, ea 1 , ee $1, \mathrm{r} 2, \ldots$ The format-based node set contained a code list of $\mathrm{r} 1, \mathrm{r} 2$, ..., ea 1 , ea $2, \ldots$ instead. Figures 1 and 2 illustrate the navigation path of 1 participant who navigated according to the presentation format through the hypertext in both node sets. As can be easily seen, there is one ordered path from top left to bottom right for the format-based node set (shown in Figure 2), but not for the thematic node set (see Figure 1). Consequently, one can enumerate how many participants within both groups used which navigation strategy within minutes.

From 79 available $\log$ files, $73 \log$ files correspond to the format-based node set. That means that, for these log files, there was more switching back and forth between nodes that were in the proximity of the format-based node set than there was back-and-forth movement between nodes that were in the proximity of the thematic node set. Twenty-four of these $73 \mathrm{log}$ files display switches exclusively between rule nodes. The other $6 \log$ files correspond to the thematic node set and belong to five skimmers and one searcher, indicating that more skimmers than searchers were able to break free from the obviously predominating format-based navigation strategy.

For a quantitative analysis of data, we consulted the Overall Stats and Overall PageStats displays for the searchers and the skimmers separately. These displays show which nodes were visited and for how long.

Processing times. All the participants processed each chapter in $30 \mathrm{~min}$. Nevertheless, there were differences between the searchers and the skimmers with respect to learning outcome, indicating differences in processing too.

The means for visited nodes reveal, for both displays, that both the searchers and the skimmers visited rule nodes (in the following, indicated by the index " $\mathrm{R} ;$ " $M_{\mathrm{R}}=$ $24.2 \mathrm{~min}, S E_{\mathrm{R}}=5.9 \mathrm{~min}$ ) longer than examples and exercises nodes [in the following indicated by the index " $\mathrm{E}$ "; $M_{\mathrm{E}}=6.4 \mathrm{~min}, S E_{\mathrm{E}}=5.7 \mathrm{~min} ; F(1,36)=283.28, p<$ $\left..0001, \eta^{2}=.89\right]$. Concordant with the patterns within the Graph display, the tendency to prefer rule nodes was stronger for the searchers $\left(M_{\mathrm{RSe}}=28.3 \mathrm{~min}, S E_{\mathrm{RSe}}=3.1 \mathrm{~min}\right.$; $M_{\mathrm{ESe}}=2.4 \mathrm{~min}, S E_{\mathrm{ESe}}=2.8 \mathrm{~min}$ ) than for the skimmers $\left[M_{\mathrm{RSk}}=20.2 \mathrm{~min}, S E_{\mathrm{RSk}}=4.8 \mathrm{~min} ; M_{\mathrm{ESk}}=10.4 \mathrm{~min}\right.$, $\left.S E_{\mathrm{ESk}}=4.6 \mathrm{~min} ; F(1,36)=58.06, p<.0001, \eta^{2}=.62\right]$. Altogether, the participants spent less than 1 min navigating through the chapters and most of their time reading the content. That holds for reading thresholds up to $10 \mathrm{sec}$ and corresponds with the netlike organization of chapters and distances between nodes of a maximum of two clicks.

Visited nodes. Within the given time of $30 \mathrm{~min}$, the skimmers $\left(M_{\mathrm{Sk}}=38.6 \%, S D_{\mathrm{Sk}}=15.3 \%\right)$ visited, as was expected, more nodes than did the searchers $\left[M_{\mathrm{Se}}=23.3 \%\right.$, $\left.S D_{\mathrm{Se}}=11.8 \% ; F(1,36)=6.21, p=.02, \eta^{2}=.15\right]$.

Processed exercises. Figure 2 shows the Graph display for answered multiple choice items. Corresponding to visited examples and exercises nodes, the skimmers processed more exercises $\left(M_{\mathrm{Sk}}=28, S D_{\mathrm{Sk}}=26.2, \min _{\mathrm{Sk}}=\right.$ $\left.0, \max _{\mathrm{Sk}}=67\right)$ than did the searchers $\left[M_{\mathrm{Se}}=4, S D_{\mathrm{Se}}=\right.$ $8.4, \min _{\mathrm{Se}}=0, \max _{\mathrm{Se}}=29 ; F(1,37)=14.59, p<.001$, $\left.\eta^{2}=.28\right]$.

\section{Discussion}

For the actual study, the Chemnitz LogAnalyzer was a useful tool for identifying different navigation strategies of participants with different processing goals. The Graph display was helpful for identifying navigation paths corresponding to either a format-based node set or a thematic ordered node set. In contrast to our hypothesis, more skimmers than searchers navigated according to the thematic order of nodes through the hypertext, instead of the format-based order. Nevertheless, they visited rule nodes almost exclusively. Thus, the searchers' paths correspond more to the format-based node set than to the thematic node set. One reason for that pattern could be that the searchers skipped examples and exercises belonging to the same theme after finding the desired answers within the rule nodes for navigating to the next theme corresponding to the next question. As was expected, the skimmers navigated in accordance with either the thematic or the format-based order of nodes. Nevertheless, the number of skimmers navigating according to the thematic node set was very small.

Overall Stats and Overall PageStats displays revealed, as was expected, less visited nodes for the searchers than for the skimmers and, correspondingly, longer visiting time per node for the searchers than for the skimmers. These differences are based on the higher number of examples and exercises nodes visited by the skimmers than by the searchers.

Nevertheless, different learning outcomes for the searchers and the skimmers were seen. Results showed that detailed questions were equally well answered by the searchers and the skimmers but that there was higher per- 
formance in application tasks by the skimmers than by the searchers. This seems to be based on different navigation behavior during the processing of the hypertext chapters. These differences correspond to comparable number and visiting times for rule nodes but different values for examples and exercises nodes. Visiting more exercises and examples for longer times could be one reason for the higher performance in application tasks, after processing, by the skimmers.

Another explanation could be that the skimmers quickly flipped through the nodes. Nevertheless, there are some arguments against that assumption as an explanation for the overall difference between the skimmers and the searchers in this study. First, the skimmers and the searchers spent most of their time reading the hypertext, and not navigating through the hypertext. Second, most of the participants preferred processing rule nodes, instead of examples and exercise nodes, for both the skimmers and the searchers. Third, performance on answering detailed questions was comparatively high for the skimmers and the searchers. And fourth, the skimmers, but not the searchers, improved their performance on application tasks by processing the chapter. Altogether, both the skimmers and the searchers processed the grammar chapters in order to understand their content, instead of quickly flipping through the nodes. Nevertheless, flipping could be a useful strategy for learners who search for very special details within a hypertext systems - for example, a date of a historical event.

\section{GENERAL DISCUSSION}

For computer-based studies, log files are compiled as raw data that cannot be easily imported into standard statistical software and that are hard to manage for a first and quick overview. Current tools such as STRATDYN, Scientific LogAnalyzer, and LOGPAT bridge that gap by analyzing navigation patterns from log files and visualizing navigation paths based on individual navigation behavior.

The Chemnitz LogAnalyzer provides further useful tools for a primary analysis and visualization of individual or group-specific navigation strategies. It can also provide a good overview of behavior, as well as a first analysis serving as the basis for a more detailed analysis in a statistics software package.

We plan in the future and have already started to implement two more features of the program. First, we have begun to integrate log files that provide data not just for 1 participant, but for many, as in many server-generated $\log$ files. Second, the option of an automatic generation of path descriptions based on the node orders for users would be desirable, and then one would have only to arrange nodes or questions, instead of generating the complete file.

\section{REFERENCES}

Berendt, B., \& Brenstein, E. (2001). Visualizing individual differences in Web navigation: STRATDYN, a tool for analyzing navigation patterns. Behavior Research Methods, Instruments, \& Computers, 33, 243-257.

Brunstein, A., \& Krems, J. F. (2003). Goal specificity and learning from educational hypertext. In Y.-F. R. Chen, L. Kovács, \& S. Lawrence (Eds.), WWW2003 conference proceedings: Alternate paper tracks (pp. 133-137). Budapest: Amulett'98 Kft.

Brunstein, A., \& Krems, J. F. (2005). Einfluss des Bearbeitungsziels auf die Strategiewahl beim hypertextgestützten Lernen. Pädagogische Psychologie/German Journal of Educational Psychology, 19, 39-48.

Brunstein, A., WanieK, J., Naumann, A., \& Krems, J. F. (2002). Learning grammar with adaptive hypertexts: Reading or searching? In P. De Bra, P. Brusilovsky, \& R. Conejo (Eds.), Adaptive hypermedia and adaptive Web-based systems (pp. 372-375). Berlin: Springer-Verlag.

DEE-LuCAS, D. (1996). Effects of overview structure on study strategies and text representations for instructional hypertext. In J.-F. Rouet, J. J. Levonen, A. P. Dillon, \& R. J. Spiro (Eds.), Hypertext and cognition (pp. 73-107). Mahwah, NJ: Erlbaum.

DeE-Lucas, D., \& LARKIN, J. H. (1999). Hypertext segmentation and goal compatibility: Effects on study strategies and learning. Journal of Educational Multimedia \& Hypermedia, 8, 279-313.

Foltz, P. W. (1996). Comprehension, coherence, and strategies in hypertext and linear text. In J.-F. Rouet, J. J. Levonen, A. P. Dillon, \& R. J. Spiro (Eds.), Hypertext and cognition (pp. 109-136). Mahwah, NJ: Erlbaum.

Hahn, A., Reich, S., \& Schmied, J. (2000). Adaptive and intelligent technologies for Web-based education. Corpus Linguistics \& Linguistic Theory, 33, 131-140.

Naumann, A., Brunstein, A., \& Krems, J. F. (2005). DEWEX: A system for designing and conducting Web-based experiments. Unpublished manuscript.

ReIPS, U.-D., \& Stieger, S. (2004). Scientific LogAnalyzer: A Webbased tool for analyses of server log files in psychological research. Behavior Research Methods, Instruments, \& Computers, 36, 304 311.

Richter, T., Naumann, J., \& Noller, S. (2003). LOGPAT: A semiautomatic way to analyze hypertext navigation behavior. Swiss Journal of Psychology, 62, 113-120.

WiLEY, J. (2001). Supporting understanding through task and browser design. In J. D. Moore \& K. Stenning (Eds.), Proceedings of the Twenty-Third Annual Conference of the Cognitive Science Society (pp. 1164-1169). Mahwah, NJ: Erlbaum.

(Manuscript received November 15, 2004; revision accepted for publication May 10, 2005.) 